\title{
Force methods for the two-relaxation-times lattice Boltzmann
}

\author{
Bart Postma (1)* \\ University of Groningen, Nijenborgh 9, 9747 AG Groningen, The Netherlands \\ Goncalo Silva ${ }^{\dagger}$ \\ Department of Mechanical Engineering, LAETA, IDMEC, IST, University of Lisbon, Avenida Rovisco Pais 1, 1049-001 Lisbon, Portugal
}

(Received 16 May 2020; revised 26 October 2020; accepted 19 November 2020; published 28 December 2020)

\begin{abstract}
The two-relaxation-times collision benefits the steady lattice Boltzmann method by yielding viscosityindependent numerical errors. We present in an intuitive way how to incorporate popular force methods into the two-relaxation-times collision. We subsequently rewrite force methods into a generic equation to reveal commonalities and differences. We prove that force methods with a second-order velocity moment of the force break the viscosity independence. A force method with only a first-order velocity moment of the force averts this breakage. We validate our proof numerically.
\end{abstract}

DOI: 10.1103/PhysRevE.102.063307

\section{VISCOSITY DEPENDENCE OF THE LATTICE BOLTZMANN METHOD}

Consider two steady incompressible fluid flows that are physically similar by having a similar Reynolds number. In addition, the first flow has its Reynolds number composed with kinematic viscosity $v_{1}$, the second flow has its Reynolds number composed with kinematic viscosity $v_{2} \neq v_{1}$. Simulate these two flows numerically with the lattice Boltzmann method $[1,2]$ using the common single-relaxation-time (SRT) collision $[3,4]$. The simulations will yield two different numerical errors, despite their flows being physically similar. Why? Because with SRT, kinematic viscosity $v$ scales the spatial discretization error [5,6]. That $v$ scales the spatial discretization error, and not merely scales the strain rate, results in unphysical behavior. Consequently, either a small or a large $v$ can result in large numerical errors.

The spatial discretization error can be rendered viscosityindependent with the two-relaxation-times (TRT) collision $[5,6]$. With TRT, user-specified constant $\Lambda$ scales the spatial discretization error. TRT's viscosity independence can break if you incorporate additional functionality into TRT, e.g., many boundary conditions break TRT's viscosity independence [6-8]. Incorporating force methods into TRT has largely been neglected, in contrast to SRT [9-15]. In this paper, first we demonstrate how to incorporate popular force methods into TRT. Second, we prove and numerically validate which force methods break TRT's viscosity independence. Thereby this paper rigorously evaluates force methods for TRT.

\section{NOMENCLATURE}

Before we commence we introduce the nomenclature of this paper, which is a nomenclature common in the TRT

\footnotetext{
*bart.postma@gmx.com

†goncalo.nuno.silva@gmail.com
}

literature $[5,6]$. Here units are specified in lattice units. The lattice Boltzmann equation is formulated as

$$
f_{q}\left(\vec{x}+\vec{c}_{q}, t+1\right)=f_{q}(\vec{x}, t)+\Omega_{q}(\vec{x}, t),
$$

with lattice quantities subscripted with a $q: \vec{c}_{q}$ being the $q$ th velocity link, $f_{q}$ is the distribution function, and $\Omega_{q}$ is the collision operator. The SRT collision is formulated as

$$
\begin{aligned}
\Omega_{q}^{\mathrm{SRT}} & =-\frac{1}{\tau}\left(f_{q}(\vec{x}, t)-\left\{e_{q}[\rho(\vec{x}, t), \vec{u}(\vec{x}, t)]+S_{q}(\vec{x}, t)\right\}\right), \\
e_{q}(\rho, \vec{u}) & =t_{q}\left(P+\hat{\rho} u_{q}+\frac{3}{2} \hat{\rho} u_{q}^{2}-\frac{1}{2} \hat{\rho} \vec{u}^{2}\right),
\end{aligned}
$$

with $e_{q}$ being the equilibrium distribution function, $t_{q}$ is the weight, $S_{q}$ is an external source with a force density $\vec{F}$, and $u_{q}=\vec{c}_{q} \cdot \vec{u}$. The relaxation time is $\tau=3 v+\frac{1}{2}$. The macroscopic quantities are as follows: mass density $\rho=$ $\sum f_{q}$, pressure $P=\rho c_{s}^{2}$ with sound speed $c_{s}=\sqrt{1 / 3}$, velocity $\vec{u}=\left(\sum \vec{c}_{q} f_{q}+\vec{F} / 2\right) / \hat{\rho}, \hat{\rho}=\rho$ for the conventional compressible model, and $\hat{\rho}=\rho_{0}$ for He and Luo's incompressible model with constant $\rho_{0}[16]$.

The TRT collision is formulated as

$$
\begin{aligned}
\Omega_{q}^{\mathrm{TRT}} & =\Omega_{q}^{+}(\vec{x}, t)+\Omega_{q}^{-}(\vec{x}, t), \\
\Omega_{q}^{+} & =-\frac{1}{\tau^{+}}\left(f_{q}^{+}(\vec{x}, t)-\left\{e_{q}^{+}[\rho(\vec{x}, t), \vec{u}(\vec{x}, t)]+S_{q}^{+}(\vec{x}, t)\right\}\right), \\
\Omega_{q}^{-} & =-\frac{1}{\tau^{-}}\left(f_{q}^{-}(\vec{x}, t)-\left\{e_{q}^{-}[\rho(\vec{x}, t), \vec{u}(\vec{x}, t)]+S_{q}^{-}(\vec{x}, t)\right\}\right), \\
e_{q}^{+}(\rho, \vec{u}) & =\frac{1}{2}\left(e_{q}+e_{\bar{q}}\right)=t_{q}\left(P+\frac{3}{2} \hat{\rho} u_{q}^{2}-\frac{1}{2} \hat{\rho} \vec{u}^{2}\right), \\
e_{q}^{-}(\rho, \vec{u}) & =\frac{1}{2}\left(e_{q}-e_{\bar{q}}\right)=t_{q} \hat{\rho} u_{q}, \\
f_{q}^{+} & =\frac{1}{2}\left(f_{q}+f_{\bar{q}}\right) \quad \text { and } \quad S_{q}^{+}=\frac{1}{2}\left(S_{q}+S_{\bar{q}}\right), \\
f_{q}^{-} & =\frac{1}{2}\left(f_{q}-f_{\bar{q}}\right) \quad \text { and } S_{q}^{-}=\frac{1}{2}\left(S_{q}-S_{\bar{q}}\right) .
\end{aligned}
$$


TABLE I. External source term $S_{q}$ of four force methods for SRT.

Buick and Greated [12]:

Guo et al. [13]:

Kupershtokh [14]:

Shan and Chen [15]:

$$
\begin{aligned}
& S_{q}=\left(\tau-\frac{1}{2}\right) t_{q} F_{q} \\
& S_{q}=\underbrace{\left(\tau-\frac{1}{2}\right) t_{q}\left(F_{q}\right.}_{\text {Buick }}+3 u_{q} F_{q}-\vec{u} \cdot \vec{F}) \\
& S_{q}=\underbrace{\left(\tau-\frac{1}{2}\right) t_{q}\left(F_{q}+3 u_{q} F_{q}-\vec{u} \cdot \vec{F}\right)}_{\text {Guo }}+\frac{t_{q}}{8 \hat{\rho}}\left(3 F_{q}^{2}-\vec{F}^{2}\right) \\
& S_{q}=\underbrace{\left(\tau-\frac{1}{2}\right) t_{q}\left(F_{q}+3 u_{q} F_{q}-\vec{u} \cdot \vec{F}\right)+\left(\frac{1}{4}\right.}_{\text {Kupershtokh }}-\tau+\tau^{2}) \frac{t_{q}}{2 \hat{\rho}}\left(3 F_{q}^{2}-\vec{F}^{2}\right)
\end{aligned}
$$

TRT decomposes an arbitrary lattice quantity $\psi$ into a symmetric part $\psi^{+}=\frac{1}{2}\left(\psi_{q}+\psi_{\bar{q}}\right)$ and an antisymmetric part $\psi^{-}=\frac{1}{2}\left(\psi_{q}-\psi_{\bar{q}}\right)$. Here $\bar{q}$ denotes the opposite link of $q$, consequently $\vec{c}_{\bar{q}}=-\vec{c}_{q}$. Relaxation time $\tau^{+}$relaxes $f_{q}^{+}$to $e_{q}^{+}$, relaxation time $\tau^{-}$relaxes $f_{q}^{-}$to $e_{q}^{-}$. The user-specified constant that scales the spatial discretization error is defined as $\Lambda=\Lambda^{+} \Lambda^{-}=\left(\tau^{+}-\frac{1}{2}\right)\left(\tau^{-}-\frac{1}{2}\right)$, where $\tau^{+}=3 v+\frac{1}{2}$ and consequently $\tau^{-}=\frac{\Lambda}{3 v}+\frac{1}{2}$. Finally, three convenience variables are $u_{\bar{q}}=\vec{c}_{\bar{q}} \cdot \vec{u}, F_{q}=\vec{c}_{q} \cdot \vec{F}$, and $F_{\bar{q}}=\vec{c}_{\bar{q}} \cdot \vec{F}$.

\section{INCORPORATING FORCE METHODS}

We incorporate four popular force methods [12-15] into TRT. These force methods also form a good test set, because they significantly differ among each other, either in form or in viscosity dependence, as shown later. All four methods discretize the force up to second order in space and time to avoid discrete lattice artifacts. Force methods with first-order space-time discretizations are inferior $[1,9,13]$, and therefore disregarded.

To compare the force methods on an equal footing, we rewrite Kupershtokh's [14] and Shan and Chen's [15] SRT formulation (Table I). These two force methods use in our paper $\vec{u}=\left(\sum \vec{c}_{q} f_{q}+\vec{F} / 2\right) / \hat{\rho}$ instead of the original $\vec{u}=$ $\left(\sum \vec{c}_{q} f_{q}\right) / \hat{\rho}$. Shan and Chen's [15] force method is further rewritten to a form using an external source term $S_{q}$ (Appendix A). From our SRT formulations we can intuitively map to TRT formulations (Table II). And from our TRT formulations we can easily deduce a generic TRT force equation for the remainder of our paper,

$$
\begin{aligned}
& S_{q}^{+}=B t_{q}\left(3 u_{q} F_{q}-\vec{u} \cdot \vec{F}\right)+C \frac{t_{q}}{2 \hat{\rho}}\left(3 F_{q}^{2}-\vec{F}^{2}\right), \\
& S_{q}^{-}=\Lambda^{-} t_{q} F_{q} .
\end{aligned}
$$

With

$\begin{array}{lll} & B & C \\ \text { Buick and Greated [12]: } & 0 & 0 \\ \text { Guo } \text { et al. [13]: } & \Lambda^{+} & 0 \\ \text { Kupershtokh [14]: } & \Lambda^{+} & \frac{1}{4} \\ \text { Shan and Chen [15]: } & \Lambda^{+} & \Lambda^{+^{2}},\end{array}$

where $\Lambda^{+}=3 v$. A generic force equation for SRT would consist of $S_{q}=S_{q}^{+}+S_{q}^{-}$and $\Lambda^{-}=\Lambda^{+}$.

\section{DERIVING FORCE ERRORS}

Next we derive the force errors that TRT with Eq. (1) appends to the well-known Navier-Stokes fluid flow equations. Our derivation assumes a flow is steady and incompressible, which is customary in the TRT literature. Steady, because time-discretization errors cannot be rendered viscosity-independent with TRT. Incompressible, because $\hat{\rho}=\rho_{0}$ reduces compressibility errors with steady flows $[17,18]$ and simplifies the mathematics. Our derivation employs the steady-state ansatz derived by Ginzburg [19]. Ginzburg's ansatz, compared to the traditional ChapmanEnskog expansion [20], relies on less assumptions and shortens the derivation. The ansatz states

$$
\begin{aligned}
& \Omega_{q}^{+}=\partial_{q}\left(e_{q}^{-}+S_{q}^{-}\right)-\Lambda^{-} \partial_{q}^{2}\left(e_{q}^{+}+S_{q}^{+}\right)+O\left(\epsilon^{3}\right), \\
& \Omega_{q}^{-}=\partial_{q}\left(e_{q}^{+}+S_{q}^{+}\right)-\Lambda^{+} \partial_{q}^{2}\left(e_{q}^{-}+S_{q}^{-}\right)+O\left(\epsilon^{3}\right),
\end{aligned}
$$

\begin{tabular}{|c|c|c|}
\hline Buick and Greated [12]: & $\begin{aligned} S_{q}^{+} & =\frac{1}{2}\left[S_{q}\left(\tau^{+}\right)+S_{\bar{q}}\left(\tau^{+}\right)\right] \\
& =\Lambda^{+}\left(\frac{1}{2} t_{q} F_{q}+\frac{1}{2} t_{\bar{q}} F_{\bar{q}}\right) \\
& =0\end{aligned}$ & $\begin{aligned} S_{q}^{-} & =\frac{1}{2}\left[S_{q}\left(\tau^{-}\right)-S_{\bar{q}}\left(\tau^{-}\right)\right] \\
& =\Lambda^{-}\left(\frac{1}{2} t_{q} F_{q}-\frac{1}{2} t_{\bar{q}} F_{\bar{q}}\right) \\
& =\Lambda^{-} t_{q} F_{q}\end{aligned}$ \\
\hline Guo et al. [13]: & $S_{q}^{+}=\Lambda^{+} t_{q}\left(3 u_{q} F_{q}-\vec{u} \cdot \vec{F}\right)$ & $S_{q}^{-}=\Lambda^{-} t_{q} F_{q}$ \\
\hline Kupershtokh [14]: & $S_{q}^{+}=\Lambda^{+} t_{q}\left(3 u_{q} F_{q}-\vec{u} \cdot \vec{F}\right)+\frac{t_{q}}{8 \hat{\rho}}\left(3 F_{q}^{2}-\vec{F}^{2}\right)$ & $S_{q}^{-}=\Lambda^{-} t_{q} F_{q}$ \\
\hline Shan and Chen [15]: & $S_{q}^{+}=\Lambda^{+} t_{q}\left(3 u_{q} F_{q}-\vec{u} \cdot \vec{F}\right)+\Lambda^{+^{2}} \frac{t_{q}}{2 \hat{\rho}}\left(3 F_{q}^{2}-\vec{F}^{2}\right)$ & $S_{q}^{-}=\Lambda^{-} t_{q} F_{q}$ \\
\hline
\end{tabular}

where $\partial_{q}=c_{q \alpha} \partial_{\alpha}$ (Greek indices denote Einstein's summation convention), $\partial_{q}^{2}=c_{q \alpha} c_{q \beta} \partial_{\alpha \beta}$, and $\epsilon$ is a small perturbation parameter. To recover the steady incompressible Navier-Stokes mass equation, take the zeroth-order velocity moment of

TABLE II. External source terms $S_{q}^{+}$and $S_{q}^{-}$of four force methods for TRT. 
Eq. (2a),

$$
\begin{aligned}
\sum \Omega_{q}^{+}= & \sum c_{q \alpha} \partial_{\alpha}\left(e_{q}^{-}+S_{q}^{-}\right)-\Lambda^{-} \sum c_{q \alpha} c_{q \beta} \partial_{\alpha \beta}\left(e_{q}^{+}+S_{q}^{+}\right) \\
0= & \sum t_{q} c_{q \alpha} c_{q \beta} \partial_{\alpha}\left(\rho_{0} u_{\beta}+\Lambda^{-} F_{\beta}\right) \\
& -\Lambda^{-} \sum t_{q} c_{q \alpha} c_{q \beta} \partial_{\alpha \beta}(P \\
& +c_{q \gamma} c_{q \delta} \frac{3}{2} \rho_{0} u_{\gamma} u_{\delta}-\frac{1}{2} \rho_{0} u_{\gamma} u_{\gamma} \\
& +c_{q \gamma} c_{q \delta} B 3 u_{\gamma} F_{\delta}-B u_{\gamma} F_{\gamma} \\
& \left.+c_{q \gamma} c_{q \delta} C \frac{3}{2 \rho_{0}} F_{\gamma} F_{\delta}-C \frac{1}{2 \rho_{0}} F_{\gamma} F_{\gamma}\right) .
\end{aligned}
$$

The zero on the left-hand side indicates no mass source is present. Substitute lattice symmetries $\sum t_{q} c_{q \alpha} c_{q \beta}=\delta_{\alpha \beta}$ and $\sum t_{q} c_{q \alpha} c_{q \beta} c_{q \gamma} c_{q \delta}=\frac{1}{3}\left(\delta_{\alpha \beta} \delta_{\gamma \delta}+\delta_{\alpha \gamma} \delta_{\beta \delta}+\delta_{\alpha \delta} \delta_{\beta \gamma}\right)$ into the equation, and work out to recover

$$
\begin{gathered}
\rho_{0} \underbrace{\partial_{\alpha} u_{\alpha}}_{\begin{array}{c}
\text { solenoidal } \\
\text { velocity }
\end{array}}+\frac{\Lambda}{3 v} \underbrace{\left[\partial_{\alpha} F_{\alpha}-\partial_{\alpha \alpha} P-\rho_{0} \partial_{\alpha \beta}\left(u_{\alpha} u_{\beta}\right)\right]}_{\text {pressure Poisson }} \\
\quad=B \frac{\Lambda}{3 v} 2 \partial_{\alpha \beta}\left(u_{\alpha} F_{\beta}\right)+C \frac{\Lambda}{3 v} \frac{1}{\rho_{0}} \partial_{\alpha \beta}\left(F_{\alpha} F_{\beta}\right) .
\end{gathered}
$$

To recover the steady incompressible Navier-Stokes momentum equation, take the first-order velocity moment of Eq. (2b),

$$
\begin{aligned}
\sum c_{q \alpha} \Omega_{q}^{-}= & \sum c_{q \alpha} c_{q \beta} \partial_{\beta}\left(e_{q}^{+}+S_{q}^{+}\right) \\
& -\Lambda^{+} \sum c_{q \alpha} c_{q \beta} c_{q \gamma} \partial_{\beta \gamma}\left(e_{q}^{-}+S_{q}^{-}\right) \\
F_{\alpha}= & \sum t_{q} c_{q \alpha} c_{q \beta} \partial_{\beta}(P \\
& +c_{q \gamma} c_{q \delta} \frac{3}{2} \rho_{0} u_{\gamma} u_{\delta}-\frac{1}{2} \rho_{0} u_{\gamma} u_{\gamma} \\
& +c_{q \gamma} c_{q \delta} B 3 u_{\gamma} F_{\delta}-B u_{\gamma} F_{\gamma} \\
& +c_{q \gamma} c_{q \delta} C \frac{3}{2 \rho_{0}} F_{\gamma} F_{\delta}-C \frac{1}{2 \rho_{0}} F_{\gamma} F_{\gamma} \\
& \left.-c_{q \gamma} c_{q \delta} \partial_{\gamma} \Lambda^{+} \rho_{0} u_{\delta}-c_{q \gamma} c_{q \delta} \partial_{\gamma} \Lambda F_{\delta}\right) .
\end{aligned}
$$

Use lattice symmetries again, to recover

$$
\begin{aligned}
\partial_{\alpha} P & +\rho_{0} \partial_{\beta}\left(u_{\alpha} u_{\beta}\right)-\rho_{0} v \partial_{\beta}\left(\partial_{\alpha} u_{\beta}+\partial_{\beta} u_{\alpha}+\delta_{\alpha \beta} \partial_{\gamma} u_{\gamma}\right)-F_{\alpha} \\
= & \frac{\Lambda}{3} \partial_{\beta}\left(\partial_{\alpha} F_{\beta}+\partial_{\beta} F_{\alpha}+\delta_{\alpha \beta} \partial_{\gamma} F_{\gamma}\right) \\
& \quad-B \partial_{\beta}\left(u_{\alpha} F_{\beta}+u_{\beta} F_{\alpha}\right)-C \frac{1}{\rho_{0}} \partial_{\beta}\left(F_{\alpha} F_{\beta}\right) .
\end{aligned}
$$

Left-hand sides of Eqs. (3) and (4) contain the targeted steady incompressible Navier-Stokes equations, right-hand sides contain the force errors. The targeted equations are still expressed in nondimensional lattice units. We rewrite the targeted equations into nondimensional physical units to maintain the "similarity principle" of a fluid flow. To that end, we introduce the nondimensional quantities

$$
\begin{gathered}
u^{*}=\frac{u}{u_{0}}, \quad P^{*}=\frac{P}{\rho_{0} u_{0}^{2}}, \quad F^{*}=F \frac{l_{0}}{\rho_{0} u_{0}^{2}}, \\
\partial_{\alpha}^{*}=l_{0} \partial_{\alpha}, \quad \operatorname{Re}=\frac{u_{0} l_{0}}{v},
\end{gathered}
$$

where $l_{0}$ is a characteristic length, or numerically viewed the spatial resolution, $u_{0}$ is a characteristic speed, and $\mathrm{Re}$ is the Reynolds number. Equations (3) and (4) then become

$$
\begin{aligned}
\partial_{\alpha}^{*} u_{\alpha}^{*} & +\frac{1}{l_{0}^{2}} \operatorname{Re} \frac{\Lambda}{3}\left[\partial_{\alpha}^{*} F_{\alpha}^{*}-\partial_{\alpha \alpha}^{*} P^{*}-\partial_{\alpha \beta}^{*}\left(u_{\alpha}^{*} u_{\beta}^{*}\right)\right] \\
& =B \underbrace{\frac{1}{l_{0}^{4}} \operatorname{Re}^{2} \frac{\Lambda}{3} 2 \partial_{\alpha \beta}^{*}\left(u_{\alpha}^{*} F_{\beta}^{*}\right)}_{\epsilon_{B}^{(m)}}+C \underbrace{v^{2} \frac{1}{l_{0}^{6}} \operatorname{Re}^{3} \frac{\Lambda}{3} \partial_{\alpha \beta}^{*}\left(F_{\alpha}^{*} F_{\beta}^{*}\right)}_{\epsilon_{C}^{(m)}},
\end{aligned}
$$

and

$$
\begin{gathered}
\partial_{\alpha}^{*} P^{*}+\partial_{\beta}^{*}\left(u_{\alpha}^{*} u_{\beta}^{*}\right)-\frac{1}{\operatorname{Re}} \partial_{\beta}\left(\partial_{\alpha}^{*} u_{\beta}^{*}+\partial_{\beta}^{*} u_{\alpha}^{*}+\delta_{\alpha \beta} \partial_{\gamma}^{*} u_{\gamma}^{*}\right)-F_{\alpha}^{*} \\
=\underbrace{\frac{1}{l_{0}^{2}} \frac{\Lambda}{3} \partial_{\beta}^{*}\left(\partial_{\alpha}^{*} F_{\beta}^{*}+\partial_{\beta}^{*} F_{\alpha}^{*}+\delta_{\alpha \beta} \partial_{\gamma}^{*} F_{\gamma}^{*}\right)}_{\epsilon_{A}^{(j)}} \\
-B \underbrace{v \frac{1}{l_{0}^{2}} \operatorname{Re} \partial_{\beta}^{*}\left(u_{\alpha}^{*} F_{\beta}^{*}+u_{\beta}^{*} F_{\alpha}^{*}\right)}_{\epsilon_{B}^{(j)}}-C \underbrace{C v^{2} \frac{1}{l_{0}^{4}} \operatorname{Re}^{2} \partial_{\beta}^{*}\left(F_{\alpha}^{*} F_{\beta}^{*}\right)}_{\epsilon_{C}^{(j)}} .
\end{gathered}
$$

A variant of Eq. (7), one without truncation errors, has been derived by Silva in a succeeding work [21]. His equation is however limited to $A \epsilon_{A}^{(j)}$ and $B \epsilon_{B}^{(j)}$ type force errors and steady channel flows.

Use Eqs. (6) and (7) to assess the force errors produced by each force method. Buick and Greated's single force error $\epsilon_{A}^{(j)}$ stems from spatially discretizing the force. This force error decreases quadratically when spatial resolution $l_{0}$ increases and scales linearly with the constant $\Lambda$. Therefore, this force error preserves TRT's viscosity independence. As a side remark, Eqs. (6) and (7) also imply Buick and Greated is a proper choice for steady incompressible SRT, even though $\epsilon_{A}^{(j)}$ then becomes viscosity-dependent, you do dispose of $B \epsilon_{B}$ and $C \epsilon_{C}$, corroborated by Ref. [10]. Guo et al.'s characteristic force errors $3 v \epsilon_{B}^{(m)}$ and $3 v \epsilon_{B}^{(j)}$ scale with $\frac{1}{l_{0}^{4}}$ and $\frac{1}{l_{0}^{2}}$. Both force errors scale with $v^{2}$. Kupershtokh's characteristic force errors $\frac{1}{4} \epsilon_{C}^{(m)}$ and $\frac{1}{4} \epsilon_{C}^{(j)}$ scale with $\frac{1}{l_{0}^{6}}$ and $\frac{1}{l_{0}^{4}}$. So particularly $\frac{1}{4} \epsilon_{C}^{(m)}$ decreases rapidly when you increase spatial resolution. Both force errors scale with $v^{2}$. Lastly, Shan and Chen's characteristic force errors $9 v^{2} \epsilon_{C}^{(m)}$ and $9 v^{2} \epsilon_{C}^{(j)}$ scale with $v^{4}$, which means these force errors increase rapidly when you increase viscosity. The force errors added by Guo et al., Kupershtokh, and Shan and Chen are viscosity-dependent, thereby they break TRT's viscosity independence. They stem from $S_{q}^{+}$, i.e., from the second-order velocity moment of the force. Guo et al., Kupershtokh, and Shan and Chen deliberately introduce $B \epsilon_{B}$, because they focus on unsteady flows, in which $B \epsilon_{B}$ 
cancels a time-dependent error. In steady flows however, this time-dependent error is not there to be canceled, causing $B \epsilon_{B}$ to subsist. They disregard $\epsilon_{A}$ and $\epsilon_{C}$ because generally $\vec{F} \propto \epsilon$ and $\partial_{\alpha} \propto \epsilon$, rendering $\epsilon_{A} \propto \epsilon^{3}$ and $\epsilon_{C} \propto \epsilon^{3}$ beyond the studied second-order perturbation expansion. Still, in certain cases $\epsilon_{A}$ and $\epsilon_{C}$ become relevant, as shown in Refs. [19,22] and in our numerical results.

\section{NUMERICAL VALIDATION}

\section{A. Setup}

To validate Eqs. (6) and (7) numerically, requires a steady incompressible flow that renders the force errors nonzero, or at least the viscosity-dependent force errors $\left(B \epsilon_{B}\right.$ and $\left.C \epsilon_{C}\right)$ nonzero. Preferably the flow also has an analytic solution to compare with. These requirements are fulfilled by the fourroll mill and by a variant of the Poiseuille flow (Appendix B) [23]. The four-roll mill comprises a periodic square domain with four counter-rotating vortices in the corners. Its analytic solution in nondimensional physical units comprises

$$
\begin{aligned}
& \vec{u}^{*}\left(x^{*}, y^{*}\right)=\left[\begin{array}{r}
\sin \left(2 \pi k_{x} x^{*}\right) \cos \left(2 \pi k_{y} y^{*}\right) \\
-\cos \left(2 \pi k_{x} x^{*}\right) \sin \left(2 \pi k_{y} y^{*}\right)
\end{array}\right] \\
& \vec{F}^{*}\left(x^{*}, y^{*}\right)=\frac{8 \pi^{2}}{\operatorname{Re}} \vec{u}^{*}\left(x^{*}, y^{*}\right), \\
& P^{*}\left(x^{*}, y^{*}\right)=\frac{1}{4}\left[\cos \left(4 \pi k_{x} x^{*}\right)+\cos \left(4 \pi k_{y} y^{*}\right)\right]+P_{\mathrm{avg}}^{*},
\end{aligned}
$$

with $x^{*}=\frac{x}{l_{x}} \in[0,1)$ and $y^{*}=\frac{y}{l_{y}} \in[0,1)$. Wave numbers $k_{x}=k_{y}=1$ yield one vortex in each corner. Average pressure $P_{\text {avg }}^{*}$ is a constant specified by the user, often set to 1 .

Poiseuille is a channel flow, our variant features a linear force and a linear pressure gradient. Its analytic solution in nondimensional physical units comprises $u_{y}^{*}=F_{y}^{*}=0$,

$$
\begin{aligned}
& u_{x}^{*}\left(y^{*}\right)=4\left(y^{*}-y^{*^{2}}\right), \quad F_{x}^{*}\left(x^{*}\right)=\frac{8}{\operatorname{Re}} x^{*} \\
& P^{*}\left(x^{*}\right)=\frac{8}{\operatorname{Re}} \frac{l_{x}}{l_{y}}\left(\frac{1}{2} x^{*^{2}}-x^{*}+\frac{1}{3}\right)+P_{\mathrm{avg}}^{*}
\end{aligned}
$$

We employ for the inlet and outlet Kim and Pitsch's [24] periodic pressure boundary condition and for the walls the familiar half-way bounce-back boundary condition. Both boundary conditions preserve TRT's viscosity independence $[6,7]$.

We choose $\mathrm{Re}=1$ to stay within the laminar regime mandated by the analytic solutions. Default $\Lambda=\frac{1}{5}$, approximately halfway the prevalent values for $\Lambda$ of $\left\{\frac{1}{12}, \frac{1}{6}, \frac{3}{16}, \frac{1}{4}, \frac{3}{8}\right\}$. Default $v=1$, approximately halfway our test range for $v$ of $[0.01,2]$. Spatial resolution is $16 \times 16$ for the four-roll mill and $23 \times 11$ for Poiseuille, deliberately low to better reveal the discrepancies among force methods. We let all force methods output a force-corrected velocity $\vec{u}=\left(\sum \vec{c}_{q} f_{q}+\vec{F} / 2\right) / \rho_{0}$. When the simulated flows reach steady state, we measure the wellknown relative $\ell^{2}$ norm of pressure errors and velocity errors,

$$
\begin{aligned}
& \epsilon_{P}=\frac{\left\|\boldsymbol{P}_{s}-\boldsymbol{P}_{a}\right\|}{\left\|\boldsymbol{P}_{a}\right\|}=\frac{\sqrt{\sum_{\vec{x}}\left[P_{s}(\vec{x})-P_{a}(\vec{x})\right]^{2}}}{\sqrt{\sum_{\vec{x}}\left[P_{a}(\vec{x})\right]^{2}}}, \\
& \epsilon_{\vec{u}}=\frac{\left\|\boldsymbol{u}_{x, s}-\boldsymbol{u}_{x, a}\right\|+\left\|\boldsymbol{u}_{y, s}-\boldsymbol{u}_{y, a}\right\|}{\left\|\boldsymbol{u}_{x, a}\right\|+\left\|\boldsymbol{u}_{y, a}\right\|},
\end{aligned}
$$

where $s$ subscripts simulated values and $a$ analytic values.

\section{B. Results}

We start by testing how each force method depends on viscosity by plotting $\epsilon_{P}$ and $\epsilon_{\vec{u}}$ vs $v$ (Fig. 1). When increasing $v$, we proportionally increase $u_{0}$ to keep the Reynolds number constant. The straight horizontal lines of Buick and Greated in all plots imply Buick and Greated does not depend on viscosity, as predicted by Eqs. (6) and (7). The steep curves of non-Buick force methods demonstrate viscosity dependence can result in large errors. Guo et al.'s errors are barely smaller than Kupershtokh's errors, which implies Kupershtokh's additional force error $\frac{1}{4} \epsilon_{C}$ is small compared to $3 v \epsilon_{B}$. Fitting polynomial curves through the errors of Guo et al. and Kupershtokh reveals that a second-order polynomial fits well, which matches the $O\left(v^{2}\right)$ predicted by Eqs. (6) and (7). Exception here is $\epsilon_{\vec{u}}$ of the four-roll mill, a third-order polynomial then fits slightly better, probably because the narrow $\epsilon_{\vec{u}}$ range hinders a reliable fit. Fitting polynomial curves through Shan and Chen's errors reveals a predicted fourth-order polynomial. Numerical experiments did show the $O\left(v^{4}\right)$ scaling only appears with very large $v$, which corroborates $\epsilon_{C}$ is small. Finally, two side remarks, not shown by the plots. A smaller or larger $\Lambda$ does not result in significant different behavior. Under rare circumstances a non-Buick curve decreases, due to $B \epsilon_{B}$ or $C \epsilon_{C}$ fortuitously canceling another error.
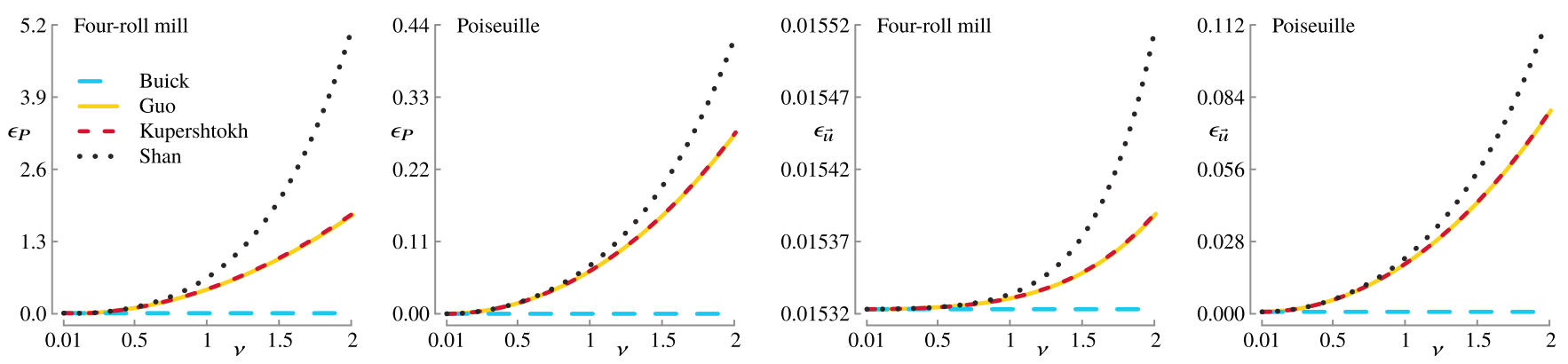

FIG. 1. Four force methods affecting the viscosity dependence of the pressure error and velocity error. $\operatorname{Re}=1$ and $\Lambda=\frac{1}{5}$. 

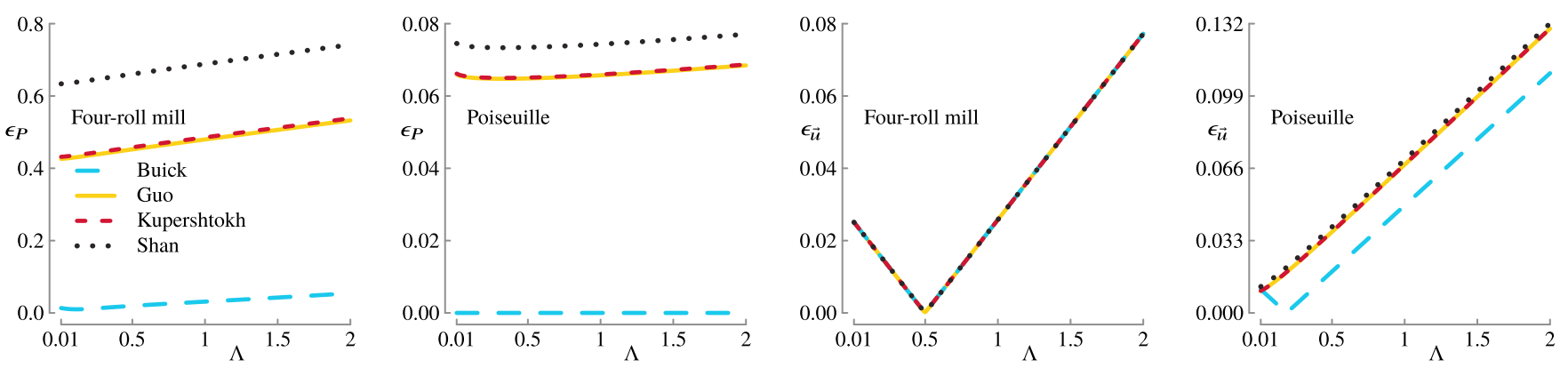

FIG. 2. Four force methods affecting the $\Lambda$ dependence of the pressure error and velocity error. $\operatorname{Re}=1$ and $v=1$.

Next, we look at how non-Buick force methods deviate from the desired behavior of Buick and Greated in plots of $\epsilon_{P}$ and $\epsilon_{\vec{u}}$ vs $\Lambda$ (Fig. 2). The TRT literature often shows this relation, because steady incompressible $\epsilon_{P}$ and $\epsilon_{\vec{u}}$ solely depend on user-specifiable $\Lambda$ and the spatial discretization error. In all four plots, non-Buick curves lie relatively close to each other. This implies $\epsilon_{C}$ is small, particularly if you consider $C_{\text {Kupershtokh }}$ and $C_{\text {Shan }}$ differ by as much as factor of $(3 \times 1)^{2} /\left(\frac{1}{4}\right)=36$. In $\epsilon_{P}$ plots, Buick and Greated's $\epsilon_{P}$ is small, because it contains no first- or second-order force errors [Eq. (6)]. Non-Buick $\epsilon_{P}$ 's by contrast, are large, due to $B \epsilon_{B}^{(m)}$ being large. In $\epsilon_{\vec{u}}$ plots, all curves scale similarly, because $B \epsilon_{B}^{(j)}$ and $C \epsilon_{C}^{(j)}$ do not depend on $\Lambda$ [Eq. (7)]. With the four-roll mill, all $\epsilon_{\vec{u}}$ curves even practically overlap. This implies the four-roll mill's $B \epsilon_{B}^{(j)}$ and $C \epsilon_{C}^{(j)}$ are negligible compared to other errors, corroborated by the small absolute differences in the four-roll mill's $\epsilon_{\vec{u}}$ vs $v$ plot (Fig. 1). As a side remark, with Buick and Greated we numerically discovered optimal $\Lambda=\frac{1}{2}$ for circular-shaped vortices. With Poiseuille, non-Buick $\epsilon_{\vec{u}}$ curves nearly overlap with each other but not with Buick and Greated's curve, implying Poiseuille's $B \epsilon_{B}^{(j)}$ is significant and $C \epsilon_{C}^{(j)}$ is not. The optimal $\Lambda=\frac{3}{16}$ of Buick and Greated equals the optimal $\Lambda$ of force-free pressure-driven
Poiseuille [6]. Indeed, the single force error $\epsilon_{A}^{(j)}$ of Buick and Greated equals 0 for a linearly increasing force. Finally, two insights, not shown by our plots with $v=1$. The optimal $\Lambda$ of a non-Buick force method is not constant but depends on $v$. The discrepancy between a non-Buick curve and Buick and Greated's curve scales with $v$.

\section{CONCLUSIONS}

From what we presented, it should be clear how to incorporate force methods into TRT and how to assess them. The equations to theoretically assess force methods agree well with the numerical results. The numerical results demonstrate force errors can become large if they are viscosity-dependent. The equations and numerical results dispel the common belief a force method always benefits from the second-order velocity moment of the force. Indeed, for steady incompressible fluids, the force method of Buick and Greated [12] is appropriate, which only contains a first-order velocity moment of the force.

\section{ACKNOWLEDGMENTS}

We thank Dr. H. Bekker and Dr. J. J. U. Postma for reviewing this work. G.S. acknowledges Fundação para a Ciência e Tecnologia through Grant No. SFRH/BPD/111228/2015 and through LAETA, Project No. UIDB/EMS/50022/2020.

\section{APPENDIX A: REWRITING THE FORCE EQUATIONS OF KUPERSHTOKH AND SHAN AND CHEN}

We rewrite the original SRT formulations of Kupershtokh [14] and Shan and Chen [15] to the SRT formulations in Table I. Let $\vec{u}=\left(\sum \vec{c}_{q} f_{q}+\vec{F} / 2\right) / \hat{\rho}$ and $e_{q}(\rho, \vec{u})=t_{q}\left(\rho c_{s}^{2}+\hat{\rho} u_{q}+\frac{3}{2} \hat{\rho} u_{q}^{2}-\frac{1}{2} \hat{\rho} \vec{u}^{2}\right)$. Rewriting Kupershtokh [14] starts by moving the external source term inside the collision,

$$
\begin{aligned}
f_{q}\left(\vec{x}+\vec{c}_{q}, t+1\right) & =f_{q}-\frac{1}{\tau}\left[f_{q}-e_{q}\left(\rho, \vec{u}-\frac{\vec{F}}{2 \hat{\rho}}\right)\right]+\underbrace{e_{q}\left(\rho, \vec{u}+\frac{\vec{F}}{2 \hat{\rho}}\right)-e_{q}\left(\rho, \vec{u}-\frac{\vec{F}}{2 \hat{\rho}}\right)}_{S_{q}^{\text {orignal }}} \\
& =f_{q}-\frac{1}{\tau}\left\{f_{q}-\left[e_{q}\left(\rho, \vec{u}-\frac{\vec{F}}{2 \hat{\rho}}\right)+\tau e_{q}\left(\rho, \vec{u}+\frac{\vec{F}}{2 \hat{\rho}}\right)-\tau e_{q}\left(\rho, \vec{u}-\frac{\vec{F}}{2 \hat{\rho}}\right)\right]\right\} . \\
e_{q}\left(\rho, \vec{u}-\frac{\vec{F}}{2 \hat{\rho}}\right) & =t_{q}\left\{c_{s}^{2} \rho+\hat{\rho}\left(\vec{c}_{q} \cdot\left[\vec{u}-\frac{\vec{F}}{2 \hat{\rho}}\right]\right)+\frac{3}{2} \hat{\rho}\left(\vec{c}_{q} \cdot\left[\vec{u}-\frac{\vec{F}}{2 \hat{\rho}}\right]\right)^{2}-\frac{1}{2} \hat{\rho}\left[\vec{u}-\frac{\vec{F}}{2 \hat{\rho}}\right]^{2}\right\},
\end{aligned}
$$


work out the terms,

$$
\begin{gathered}
\hat{\rho}\left(\vec{c}_{q} \cdot\left[\vec{u}-\frac{\vec{F}}{2 \hat{\rho}}\right]\right)=\hat{\rho} \vec{c}_{q} \cdot \vec{u}-\frac{1}{2} \vec{c}_{q} \cdot \vec{F}=\hat{\rho} u_{q}-\frac{1}{2} F_{q}, \\
\frac{3}{2} \hat{\rho}\left(\vec{c}_{q} \cdot\left[\vec{u}-\frac{\vec{F}}{2 \hat{\rho}}\right]\right)^{2}=\frac{3}{2} \hat{\rho} u_{q}^{2}-\frac{3}{2} u_{q} F_{q}+\frac{3}{8 \hat{\rho}} F_{q}^{2}, \\
-\frac{1}{2} \hat{\rho}\left[\vec{u}-\frac{\vec{F}}{2 \hat{\rho}}\right]^{2}=-\frac{1}{2} \hat{\rho} \vec{u}^{2}+\frac{1}{2} \vec{u} \cdot \vec{F}-\frac{1}{8 \hat{\rho}} \vec{F}^{2},
\end{gathered}
$$

and combine them to

$$
e_{q}\left(\rho, \vec{u}-\frac{\vec{F}}{2 \hat{\rho}}\right)=e_{q}(\rho, \vec{u})-\frac{t_{q}}{2}\left(F_{q}+3 u_{q} F_{q}-\vec{u} \cdot \vec{F}\right)+\frac{t_{q}}{8 \hat{\rho}}\left(3 F_{q}^{2}-\vec{F}^{2}\right)
$$

Analogously,

$$
e_{q}\left(\rho, \vec{u}+\frac{\vec{F}}{2 \hat{\rho}}\right)=e_{q}(\rho, \vec{u})+\frac{t_{q}}{2}\left(F_{q}+3 u_{q} F_{q}-\vec{u} \cdot \vec{F}\right)+\frac{t_{q}}{8 \hat{\rho}}\left(3 F_{q}^{2}-\vec{F}^{2}\right) .
$$

Substitute the equations of $e_{q}\left(\rho, \vec{u}-\frac{\vec{F}}{2 \hat{\rho}}\right)$ and $e_{q}\left(\rho, \vec{u}+\frac{\vec{F}}{2 \hat{\rho}}\right)$ into $f_{q}\left(\vec{x}+\vec{c}_{q}, t+1\right)$ and work out to acquire

$$
f_{q}\left(\vec{x}+\vec{c}_{q}, t+1\right)=f_{q}-\frac{1}{\tau}\left(f_{q}-\left[e_{q}(\rho, \vec{u})+\left(\tau-\frac{1}{2}\right) t_{q}\left(F_{q}+3 u_{q} F_{q}-\vec{u} \cdot \vec{F}\right)+\frac{t_{q}}{8 \hat{\rho}}\left(3 F_{q}^{2}-\vec{F}^{2}\right)\right]\right) .
$$

Next we rewrite Shan and Chen [15], starting again from the original SRT formulation,

$$
\begin{gathered}
f_{q}\left(\vec{x}+\vec{c}_{q}, t+1\right)=f_{q}-\frac{1}{\tau}\left[f_{q}-e_{q}\left(\rho, \vec{u}-\frac{\vec{F}}{2 \hat{\rho}}+\frac{\tau \vec{F}}{\hat{\rho}}\right)\right], \\
e_{q}\left(\rho, \vec{u}-\frac{\vec{F}}{2 \hat{\rho}}+\frac{\tau \vec{F}}{\hat{\rho}}\right)=t_{q}\left\{c_{s}^{2} \rho+\hat{\rho}\left(\vec{c}_{q} \cdot\left[\vec{u}-\frac{\vec{F}}{2 \hat{\rho}}+\frac{\tau \vec{F}}{\hat{\rho}}\right]\right)+\frac{3}{2} \hat{\rho}\left(\vec{c}_{q} \cdot\left[u-\frac{\vec{F}}{2 \hat{\rho}}+\frac{\tau \vec{F}}{\hat{\rho}}\right]\right)^{2}-\frac{1}{2} \hat{\rho}\left[u-\frac{\vec{F}}{2 \hat{\rho}}+\frac{\tau \vec{F}}{\hat{\rho}}\right]^{2}\right\},
\end{gathered}
$$

work out the terms,

$$
\begin{aligned}
& \hat{\rho}\left(\vec{c}_{q} \cdot\left[\vec{u}-\frac{\vec{F}}{2 \hat{\rho}}+\frac{\tau \vec{F}}{\hat{\rho}}\right]\right)=\hat{\rho} u_{q}-\frac{1}{2} F_{q}+\tau F_{q}, \\
& \frac{3}{2} \hat{\rho}\left(\vec{c}_{q} \cdot\left[\vec{u}-\frac{\vec{F}}{2 \hat{\rho}}+\frac{\tau \vec{F}}{\hat{\rho}}\right]\right)^{2}=\frac{3}{2} \hat{\rho} u_{q}^{2}+\left(\tau-\frac{1}{2}\right) 3 u_{q} F_{q}+\left(\frac{1}{8 \hat{\rho}}-\frac{\tau}{2 \hat{\rho}}+\frac{\tau^{2}}{2 \hat{\rho}}\right) 3 F_{q}^{2}, \\
&-\frac{1}{2} \hat{\rho}\left[\vec{u}-\frac{\vec{F}}{2 \hat{\rho}}+\frac{\tau \vec{F}}{\hat{\rho}}\right]^{2}=-\frac{1}{2} \hat{\rho} \vec{u}^{2}-\left(\tau-\frac{1}{2}\right) \vec{u} \cdot \vec{F}-\left(\frac{1}{8 \hat{\rho}}-\frac{\tau}{2 \hat{\rho}}+\frac{\tau^{2}}{2 \hat{\rho}}\right) \vec{F}^{2},
\end{aligned}
$$

and combine them to

$$
e_{q}\left(\rho, u-\frac{\vec{F}}{2 \hat{\rho}}+\frac{\tau \vec{F}}{\hat{\rho}}\right)=e_{q}(\rho, \vec{u})+\left(\tau-\frac{1}{2}\right) t_{q}\left(F_{q}+3 u_{q} F_{q}-\vec{u} \cdot \vec{F}\right)+\left(\frac{1}{4}-\tau+\tau^{2}\right) \frac{t_{q}}{2 \hat{\rho}}\left(3 F_{q}^{2}-\vec{F}^{2}\right) .
$$

Substitute this equation into $f_{q}\left(\vec{x}+\vec{c}_{q}, t+1\right)$ to finish with

$$
f_{q}\left(\vec{x}+\vec{c}_{q}, t+1\right)=f_{q}-\frac{1}{\tau}\left(f_{q}-\left[e_{q}(\rho, \vec{u})+\left(\tau-\frac{1}{2}\right) t_{q}\left(F_{q}+3 u_{q} F_{q}-\vec{u} \cdot \vec{F}\right)+\left(\frac{1}{4}-\tau+\tau^{2}\right) \frac{t_{q}}{2 \hat{\rho}}\left(3 F_{q}^{2}-\vec{F}^{2}\right)\right]\right) .
$$

\section{APPENDIX B: DERIVING THE EQUATIONS OF THE BENCHMARK FLOWS}

The four-roll mill is the steady version of the unsteady Taylor-Green vortex (TGV) [25] at $t=0$. The four-roll mill's analytic velocity [Fig. 3(a)] is therefore

$$
\vec{u}(x, y)=\vec{u}^{\mathrm{TGV}}(x, y, t=0)=\left[\begin{array}{l}
Q \sin (q x) \cos (r y) \\
R \cos (q x) \sin (r y)
\end{array}\right] \underbrace{D(t=0)}_{=1},
$$

with constants

$$
Q=u_{0} \sqrt{\frac{k_{y} l_{x}}{k_{x} l_{y}}}, \quad R=-u_{0} \sqrt{\frac{k_{x} l_{y}}{k_{y} l_{x}}}, \quad q=k_{x} \frac{2 \pi}{l_{x}}, \quad r=k_{y} \frac{2 \pi}{l_{y}},
$$

and with $x \in\left[0, l_{x}\right), y \in\left[0, l_{y}\right)$, and $D(t)=e^{-\left(q^{2}+r^{2}\right) v t}$. Wave numbers $k_{x}$ and $k_{y}$ control the number of vortices in the $x$ and $y$ directions. The velocity magnitude is controlled by $u_{0}$. To keep the flow steady, a force density $\vec{F}$ counterbalances the 


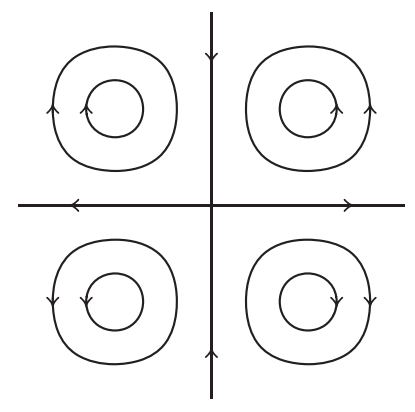

(a) Four-roll mill

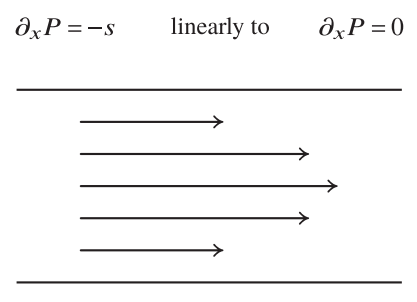

$F_{x}=0 \quad$ linearly to $\quad F_{x}=s$

(b) Poiseuille
FIG. 3. Velocity streamlines of the benchmark flows.

Taylor-Green vortex's deceleration,

$$
\vec{F}(x, y)=-\left.\rho_{0} \partial_{t} \vec{u}^{\mathrm{TGV}}\right|_{t=0}=\rho_{0}\left(q^{2}+r^{2}\right) \nu \vec{u}(x, y) .
$$

To acquire an equation for the pressure $P$, substitute $\vec{u}$ into the Navier-Stokes momentum equations, combine and rewrite the results to a Poisson equation for the pressure, finally integrate the Poisson equation to acquire

$$
P(x, y)=\frac{\rho_{0} Q^{2}}{4}\left(\cos (2 q x)+\frac{q^{2}}{r^{2}} \cos (2 r y)\right)+P_{\text {avg }},
$$

with average pressure $P_{\text {avg }}$. Use the nondimensional quantities of Eq. (5) to nondimensionalize $\vec{u}, \vec{F}$, and $P$ into $\vec{u}^{*}, \vec{F}^{*}$, and $P^{*}$ of Eq. (8) with $l_{0}=\frac{l_{x}}{k_{x}}=\frac{l_{y}}{k_{y}}$ (wavelength).
Poiseuille flow [26] has an analytic velocity of

$$
u_{x}(y)=\frac{s}{2 \rho_{0} v} y\left(l_{y}-y\right),
$$

with constant $s=F_{x}-\partial_{x} P$. Generally, the channel flow is driven by either a constant force density $F_{x}$ or a constant pressure gradient $\partial_{x} P$. Occasionally, it is driven by both a constant $F_{x}$ and a constant $\partial_{x} P$. In our Poiseuille variant, $F_{x}$ runs linearly from 0 to $s$ and $\partial_{x} P$ runs linearly from $-s$ to 0 , from $x=0$ to $x=l_{x}$ [Fig. 3(b)]. Consequently, the equations for $F_{x}$ and $P$ become

$$
\begin{aligned}
F_{x}(x) & =\frac{s}{l_{x}} x, \\
P(x) & =\int \partial_{x} P d x \\
& =\int\left(\frac{s}{l_{x}} x-s\right) d x \\
& =\frac{1}{2} \frac{s}{l_{x}} x^{2}-s x+P_{\mathrm{int}} .
\end{aligned}
$$

Integration constant $P_{\text {int }}$ can be determined by specifying an average pressure,

$$
P_{\text {avg }}=\frac{1}{l_{x}} \int_{0}^{l_{x}} P(x) d x=-\frac{1}{3} s l_{x}+P_{\text {int }} .
$$

Again use Eq. (5) to nondimensionalize $\vec{u}, \vec{F}$, and $P$ into $\vec{u}^{*}, \vec{F}^{*}$, and $P^{*}$ of Eq. (9) with $l_{0}=l_{y}$ and $u_{0}=u_{x}\left(\frac{l_{y}}{2}\right)$.
[1] T. Krüger, H. Kusumaatmaja, A. Kuzmin, O. Shardt, G. Silva, and E. M. Viggen, The Lattice Boltzmann Method: Principles and Practice, Graduate Texts in Physics (Springer, Berlin, 2017).

[2] D. A. Wolf-Gladrow, Lattice-Gas Cellular Automata and Lattice Boltzmann Models, Lecture Notes in Mathematics (Springer, Berlin, 2000).

[3] Y. H. Qian, D. d'Humières, and P. Lallemand, Europhys. Lett. 17, 479 (1992).

[4] H. Chen, S. Chen, and W. H. Matthaeus, Phys. Rev. A 45, R5339(R) (1992).

[5] D. d'Humières and I. Ginzburg, Comput. Math. Appl. 58, 823 (2009).

[6] I. Ginzburg, F. Verhaeghe, and D. d'Humières, Commun. Comput. Phys. 3, 427 (2008).

[7] G. Silva, I. Ginzburg, and L. Talon, J. Comp. Phys. 335, 50 (2017).

[8] S. Gsell, U. D’Ortona, and J. Favier, Phys. Rev. E 100, 033306 (2019).

[9] H. Huang, M. Krafczyk, and X. Lu, Phys. Rev. E 84, 046710 (2011).

[10] G. Silva and V. Semiao, Physica A 390, 1085 (2011).
[11] A. A. Mohamad and A. Kuzmin, Int. J. Heat Mass Transf. 53, 990 (2010).

[12] J. M. Buick and C. A. Greated, Phys. Rev. E 61, 5307 (2000).

[13] Z. Guo, C. Zheng, and B. Shi, Phys. Rev. E 65, 046308 (2002).

[14] A. L. Kupershtokh, in the 5th International EHD Workshop, Poitiers, France (Elsevier, New York, 2004), pp. 241-246.

[15] X. Shan and H. Chen, Phys. Rev. E 47, 1815 (1993).

[16] X. He and L.-S. Luo, J. Stat. Phys. 88, 927 (1997).

[17] P. J. Dellar, J. Comput. Phys. 190, 351 (2003).

[18] L.-S. Luo, W. Liao, X. Chen, Y. Peng, and W. Zhang, Phys. Rev. E 83, 056710 (2011).

[19] I. Ginzburg, Phys. Rev. E 77, 066704 (2008).

[20] U. Frisch, B. Hasslacher, and Y. Pomeau, Phys. Rev. Lett. 56, 1505 (1986).

[21] G. Silva, Comput. Fluids 203, 104537 (2020).

[22] D. Lycett-Brown and K. H. Luo, Phys. Rev. E 91, 023305 (2015).

[23] See Supplemental Material at http://link.aps.org/supplemental/ 10.1103/PhysRevE.102.063307 for the computer code of our numerical validation.

[24] S. H. Kim and H. Pitsch, Phys. Fluids 19, 108101 (2007).

[25] G. I. Taylor and A. E. Green, Proc. R. Soc. A 158, 499 (1937).

[26] M. Alaoui and A. Santos, Phys. Fluids A 4, 1273 (1992). 